\title{
Energy-Efficient Area Coverage in Heterogeneous Energy Wireless Sensor Networks
}

\author{
Yingchi Mao, Xiaofang Li, Feng Xu \\ College of Computer and Information Engineering, Hohai University, Nanjing, China \\ Email:yingchimao@hhu.edu.cn \\ Received April 25, 2010; revised June 18, 2010; accepted August 5, 2010
}

\begin{abstract}
Sensing coverage and energy consumption are two primary issues in wireless sensor networks. Sensing coverage is closely related to network energy consumption. The performance of a sensor network depends to a large extent on the sensing coverage, and its lifetime is determined by its energy consumption. In this paper, an energy-efficient Area Coverage protocol for Heterogeneous Energy sensor networks (ACHE) is proposed. ACHE can achieve a good performance in terms of sensing area coverage, lifetime by minimizing energy consumption for control overhead, and balancing the energy load among all nodes. Adopting the hierarchical clustering idea, ACHE selects the active nodes based on the average residual energy of neighboring nodes and its own residual energy parameters. Our simulation demonstrates that ACHE not only provide the high quality of sensing coverage, but also has the good performance in the energy efficiency. In addition, ACHE can better adapt the applications with the great heterogeneous energy capacities in the sensor networks, as well as effectively reduce the control overhead.
\end{abstract}

Keywords: Wireless Sensor Networks, Area Coverage, Heterogeneous Energy, Location-Independent

\section{Introduction}

Wireless sensor networks (WSNs) consist of tiny, lowcost, low-power tiny sensors that can communicate with each other to perform sensing and data processing cooperatively. Sensors in WSNs can cooperatively gather information from an interested region of observation and transmit it to a base station [1]. Maintaining a sufficient sensing coverage is a critical requirement of sensor networks, since sensing coverage directly determines the monitoring quality provided by sensor networks in a designated region. By turning off redundant (in term of coverage) sensor nodes, the coverage quality can be maintained and energy efficiency can be achieved at the same time.

In order to prolong the lifetime of a WSN, an effective coverage control protocol should be have the following features.

1) It should be completely distributed and self-confi gured. Each node independently makes its decision based on the local information, within a constant number of hops. Because WSN has a dynamic topology and needs to accommodate a large number of sensors, the protocols should be distributed and localized to better accommo- date a scalable architecture.

2) The working nodes should be well-distributed over the monitored field in order to balance the energy load among the sensors. Because the energy consumption of working sensors are greater than those of sleeping sensors, the poor distribution of working sensors may result in the sensing hole.

3) It should have low control overhead for coverage protocol, which can long the network lifetime.

4) It should be able to handle the heterogeneous energy capacities among the network. In real-world applications, it is unrealistic to guarantee all sensors have the same energy. For example, in environment monitoring, some nodes are active and some are sleep. They have different energy load. On the other hand, due to the different position of active nodes, they may consume different energy for collect and delivery sensed data. In addition, sensors redeployment also results in the heterogeneous energy capacities.

5) It should not dependent on the accurate location or directional information such as that obtained with the GPS and the directional antenna technology. The energy cost and system complexity may compromise the effectiveness of proposed solution as a whole. Furthermore, it 
is still a very difficult problem to estimate sensors' locations, since GPS and other complicated hardware devices consume too much energy and the costs are too high for tiny sensors [2].

To meet the above requirements, an energy-efficient location-independent area coverage protocol for heterogeneous energy sensor networks (ACHE) is proposed. Different from DELIC [3], adopting the hierarchical clustering idea, ACHE elects the working nodes based on the average residual energy of neighboring nodes and its own residual energy parameters. Simulation study demonstrates that ACHE can better adapt the application with the heterogeneous energy capacities and also effectively reduce the control overhead.

The rest of the paper is organized as follows. In Section 2, we will give a brief summary of the related work. Section 3 describes the network model. Following that, we present in Section 4 the proposed coverage protocol ACHE. Section 5 will give the simulation and evaluation results. Section 6 is the conclusion remarks.

\section{Related Work}

In [4] and [5], an approach is proposed to compute the maximal cover set. The sensor nodes in each cover set can perform independently the task of monitoring the desired area; sensor nodes in all the cover sets take turns at performing the monitoring task. The two algorithms proposed are both centralized ones, so they are not suitable for the case in which there is a large quantity of sensors.

In [6], Tian et al. propose a off-duty eligibility rule for complete sensing coverage, relying on the geographical information of sensor nodes and AOA (Arrival of Angle) obtained through the directional antenna, can reckon the coverage relation between one node and its neighbors and then select working nodes. The off-duty eligibility rule fails to consider the problem that excessive overlap may be formed so that the number of working nodes selected becomes very large to cause extra energy consumption.

In [7], it is proved that to ensure that the full sensing area coverage of a convex area also guarantee the connectivity of the active nodes, the communication range should be at least twice of the sensing range. Based on the analytical results, Zhang et al. proposed a distributed, localized density control algorithm named OGDC [7]. Wang et al. draw the same conclusion in [8], and presented a Coverage Configuration Protocol (CCP) that can provide complete coverage. Based on OGDC, Wu et al. proposed a coverage control protocol with adjustable sensing range [9]. Every working node adjusts the appropriate sensing range based on the relative position to the neighboring nodes in order to decrease the excessive overlap of sensing coverage.

The above coverage protocols have to rely on the location information of sensor nodes in reckoning sensing coverage. The following protocols do not require the location information to solve the coverage problem.

Kumar et al. adopt the Randomized Independent Scheduling (RIS) mechanism [10]. At the beginning of each round, each sensor independently decides whether to become active with probability $\mathrm{p}$ or go to sleep with probability $1-p, p$ determines the network lifetime. Obviously, RIS does not require location information and has no communication overhead. But RIS is not robust against unexpected failure before they run out of energy.

Ye et al. proposed a probing-based density control algorithm named PEAS [11]. In PEAS, a sleeping node wakes up periodically to probe its neighbors and transits to working mode if there is no working node within its probing range. Obviously, in PEAS, some nodes may die too early and the energy consumption is unbalance, which will severely affect the coverage quality.

Gao et al. propose a mathematical method, which does not rely on location information, to describe the redundancy [12]. According to this method, one sensor node can utilize the number of neighbors within its sensing range to calculate its own probability of becoming a redundant node. However, for most sensor nodes, the sensing hardware and the communicating hardware are two fully independent parts, and the communicating range is always not equal to the sensing range. Therefore, some specialized parts are needed to judge the number of neighbors within the sensing range.

Mao et al. proposed a location-independent coverage protocol DELIC [3]. DELIC adopt the hierarchical clustering mechanism for working sensor election by comparing its own residual energy to that of its neighboring nodes. However, DELIC require several iteration to determine final working sensors, which results the greater control overhead.

Although the above protocols do not need the accurate location information of sensors, they also have some limitation. In addition, they could not discuss how to effectively deal with the heterogeneous energy capacities for coverage control.

\section{Network Model}

Assume N sensor nodes are randomly and uniformly distributed over the sensing field $\boldsymbol{A}$. The sensor density is high enough that any point of $\boldsymbol{A}$ can be monitored by sensor nodes. We assume the following properties about the wireless sensor network:

1) The nodes in the network are stationary and they are left unattended after deployment. 
2) For simplicity and convenience, the sensing mode is Boolean mode.

3) All nodes should be roughly time synchronized on the order of seconds.

4) All nodes have different initial energy and the battery could not be rechargeable.

5) Nodes are location-unaware, i.e. not equipped with GPS-capable antennae.

6) Sensor's radio transceiver is capable of changing its transmission power in continuous steps to achieve different transmission ranges. Some sensors, such as the MICAZ mote, provide multiple levels of transmission power.

The first three properties are typical assumptions in general wireless sensor networks. The forth property is more suitable for the real-world application compared with the same initial energy of all nodes. The fifth property assumes the nodes are location-unaware, which can decrease the hardware cost and energy consumption of sensors. From the point of view of energy efficiency, compared with the fix transmission power, the last property can improve the energy efficiency to prolong the network lifetime.

\section{ACHE Protocol}

In a WSN, the working sensors should receive and delivery the sensed data, which results in the higher energy consumption than that of sleeping sensors. In order to balance the energy load among the sensors in a WSN, ACHE protocol involves round-operation. Each round includes two phases: Decision phase and Sensing phase, denoted by DT and ST respectively. In DT, based on the hierarchical clustering idea, ACHE selects the working nodes. First, every node exchange the information with its neighbor nodes, then every node compete the working node based on the average residual energy of neighbor nodes and its own residual energy. After decision phase, the working sensors can collect and delivery sensed data to the base station, while other sensors enter into the sleeping state.

\subsection{Adjust Appropriate Transmission Range}

In [13], we have given the theoretical analysis of the sensing area coverage property of minimal dominating set (MDS). Furthermore, we established the relationship between point coverage and area coverage in random geometric graphs.

Suppose sensor nodes follow Poisson point process of density $\lambda$ in the plane $\mathbb{R}^{2}$. According to (1), it can compute the appropriate transmission range $R t$.

$$
R t=R s-r
$$

$$
=R s-\min \left(R s, \sqrt{\left.\frac{-\ln \left(\varepsilon+(1-\varepsilon) e^{-\pi \lambda R s^{2}}\right)}{\pi \lambda}\right)}\right.
$$

For $\forall \varepsilon>0$, any minimal dominating set of $G_{t}$ (recall that $G_{t}$ is a subgraph of $G$ induced by transmission range $R t$ ) provides the same coverage as that of all sensor nodes with probability at least $1-\varepsilon$.

On the other hand, Theorem 1 proves the set of working nodes elected by ACHE must be a dominating set in Subsection 4.3. Since MDS $\subseteq$ CDS, we have Area $(M D S) \leq \operatorname{Area}(D S)$. By (1), every node can adjust the appropriate transmission range $R t$, according to the local density $\lambda$, ACHE guarantee that the working nodes can provide the high quality of sensing coverage.

With the execution of the sensor network, sensor nodes are prone to fail, the value of $\lambda$ is changing. For a single node, it is very difficult to know the change of $\lambda$. Therefore, each node determines a local node density $\lambda_{\text {local }}$ by counting the number of nodes in its $k$-hop neighbors using the maximum transmission range $R t$ max. The local node density $\lambda_{\text {local }}$ can be approximated as the network density $\lambda$ and can be adapt to variations of node density. The local node density $\lambda_{\text {local }}$ can be computed as follows:

$$
\lambda_{\text {local }}=\frac{|c|+1}{\pi\left(R t_{\text {max }}\right)^{2} k^{2}}
$$

where $|c|$ is the number of $k$-hop neighbors. The larger the value of $k$ is, the more accurate the approximation of $\lambda_{\text {local }}$ is. However, the increase of the number of messages will results in the more energy consumption. ACHE estimates the local density $\lambda_{\text {local }}$ by counting the number of nodes in its 1-hop neighbor nodes using $R t$ max .

$$
\text { Let } \varepsilon_{0}=\varepsilon+(1-\varepsilon) e^{-\pi \lambda R s^{2}} \text {. Clearly, } \varepsilon_{0} \in(0,1) \text {. Comb- }
$$

ing the (1) and (2), we have

$$
\begin{aligned}
R t & =R s-\min \left(R s, \sqrt{\frac{-\ln \varepsilon_{0}}{\pi \lambda_{\text {local }}}}\right) \\
& =R s-\min \left(R s, \sqrt{\frac{-\ln \varepsilon_{0}}{|c|+1}}\right)
\end{aligned}
$$

Therefore, each sensor node can adjust the appropriate the transmission range $R t$ by (3). The obtained sensing coverage ratio has the probability at least $1-\varepsilon$.

\subsection{Working Nodes Selection}

Definition 1: Neighborhood. For any node $S_{i}$, the neighborhood node $S_{j}$ of node $S_{i}$ is defined as $S_{j}=\left\{d\left(S_{i}, S_{j}\right) \leq R t, j \neq i\right\}$, where $d\left(S_{i}, S_{j}\right)$ denotes the distance between node $S_{i}$ and node $S_{j}$, Rt denotes the transmission range computed by (3).

In order to effectively deal with the heterogeneous energy capacities in sensor networks, it is obvious that the 
higher energy the node has, the higher probability it becomes working nodes. Meanwhile, the lower average energy of its neighborhood nodes, the higher probability it becomes working nodes. Thus, ACHE elects the working nodes considering the parameters, the average residual energy of neighborhood nodes and the node own residual energy.

In ACHE, every node requires to maintain a neighborhood table Neighbor_Table, recording the neighbors' ID, neighbors' state, and neighbors' residual energy. At the beginning of each round, every node first broadcasts an Energy_Msg message with the transmission range $R t$ to its neighboring nodes. The Energy_Msg message includes ID of the sender node, state and its residual energy. Once receiving the $\boldsymbol{E}$ nergy_Msg from its neighborring nodes, the node should update its own Neighbor_ Table. After updating its Neighbor_Table, every node $S i$ can compute the average residual energy of its neighborring nodes, $S_{i} \cdot E_{\text {avg }}$. Let

$$
S_{i} \cdot E_{\text {avg }}=\frac{\sum_{j=1}^{m} S_{j} \cdot E_{\text {residual }}}{m},(1 \leq i \leq N)
$$

where $m$ is the number of neighboring nodes of $S_{i}$, $S_{j} . E_{\text {residual }}(1 \leq j \leq m, j \neq i)$ denotes the residual energy of node $S_{j}$.

According to (4) and (5), we can obtain the time $t_{i}$ when the node $S_{i}$ broadcasts an Active_Msg message to announce node $S_{i}$ would be a working node. An $\boldsymbol{A c}$ tive_Msg message consists of ID of the sender node, and its state ACTIVE.

$$
t_{i}=k \times T \times \frac{S_{i} \cdot E_{\text {avg }}}{S_{i} \cdot E_{\text {residual }}},(1 \leq i \leq N)
$$

where $k$ is a random uniformly distributed in $[0.9,1.0] ; T$ is the time length for selecting working nodes in each round, that is $T=D T ; \mathrm{S}_{\mathrm{i}} \cdot \mathrm{E}_{\text {residual }}$ denotes the residual energy of node $S_{i}$.

From (5), it can notice that ACHE protocol uses $S_{i} \cdot E_{\text {avg }} / S_{i} \cdot E_{\text {residual }}$ as the main parameter for selecting working nodes. That is to say, the time $t_{i}$ when the node $\mathrm{Si}$ broadcasts an Active_Msg message is mainly determined by $S_{i} \cdot E_{\text {avg }} / S_{i} \cdot E_{\text {residual }}$. Compared with DELIC protocol, which is only based on its own residual energy of node to compete the working node, ACHE can better handle the heterogeneous energy capacities among the sensor nodes.

During the phase of selection working nodes, any node $S_{i}$ checks whether it receives any Active $M s g$ from its neighboring nodes before the time $t_{i}$ expires. If node $S_{i}$ gets the Active_Msg from neighbor node $S_{j}$ before the time $t_{i}$ expires, node $S_{i}$ would lose the chance to become the working node, and enter into the SLEEP state. That is to say, node $S_{i}$ is in the sensing coverage of the neighbor node $S_{j}$. If node $S_{i}$ does not receive any Active_Msg from its neighboring nodes when the time $t_{i}$ expires, which means there is no neighbor node can cover the sensing area of node $S_{i}$, node $S_{i}$ broadcasts an Active_Msg to its neighboring nodes, and becomes a working node and changes its state to ACTIVE. However, if another neighbor node $S_{k}$ also broadcasts an Active_Msg message between the time interval $\left(t_{i}-\Delta t, t_{i}+\Delta t\right)$, there maybe have several working nodes within each other's transmission range Rt. $\Delta t$ is the time interval than all neighboring nodes can receive Active_Msg message from node $S_{i}$ in the worst case. Due to the short package and limited broadcast range of Active_Msg message, $\Delta t$ is quite small. Thus, the probability that several nodes within the transmission range $R t$ are working nodes is small. Theorem 3 would prove the result in Subsection 4.3.

From the above description for ACHE, it is clearly found that there are only working nodes could broadcast Active_Msg message, and every working node only broadcast one Active_Msg message during the phase of selecting working nodes, which can greatly reduce the control overhead.

\subsection{Protocol Analysis}

ACHE is a completely distributed, self-configured sensing coverage protocol. Node decisions are based solely on local information. This section provides the theoretical analysis of ACHE for a better understanding.

Theorem 1: The set of working sensor nodes obtained by ACHE forms a dominating set. That is to say, for any sensor node $v$ in the deployed sensor set $N$, node $v$ is either in the set of working sensor nodes or one of node $v$ 's neighbor nodes is in the set of working sensor nodes.

Proof: Randomly select a sensor node $v$ in the deployed sensor set $N$. If $v$ is a working sensor node, Theorem 1 holds. For the remaining case, we will show that there exists a working neighboring sensor node of $v$. Since $v$ is not a working node, there exists at least one working node $u$ in the neighboring nodes of $v$.

Moreover, the time $t_{u}$ when node $u$ broadcasts $A c$ tive_Msg message is earlier than that of node $v$. In another words, before the time $t_{v}$ expires, $v$ has received an Active_Msg from $u$, and entered into the SLEEP state. Otherwise, once the time $t_{v}$ expires, $v$ has not received any Active_Msg from its neighboring nodes, becomes a working node, and broadcasts an Active_Msg to it neighborhood.

Clearly, for any sensor node $v$ in the deployed sensor set $N$, node $v$ is either in the set of working sensor nodes or one of node $v$ 's neighbor nodes is in the set of work- 
ing sensor nodes.

Theorem 2: $\forall \varepsilon>0$, the set of working sensor nodes obtained by ACHE can provide the complete sensing coverage with probability at least $1-\varepsilon$.

Proof: In [13], we have proved that for $\forall \varepsilon>0$, any minimal dominating set of $G_{t}\left(G_{t}\right.$ is a subgraph of $G$ induced by transmission range $R t$ based on (3)) provides the same coverage as that of all sensor nodes with probability at least $1-\varepsilon$.

In the network model, it assumes that the sensor density is high enough that any point of $\boldsymbol{A}$ can be monitored by sensor nodes. Since MDS $\subseteq$ CDS, we have $\operatorname{Area}(M D S) \leq \operatorname{Area}(D S)$. According Theorem 1, the set of working sensor nodes obtained by ACHE forms a dominating set. Therefore, $\forall \varepsilon>0$, the set of working sensor nodes obtained by ACHE can provide the complete sensing coverage with probability at least $1-\varepsilon$.

Theorem 3: In each round, the probability that several working nodes within one transmission range $R t$ is small, i.e., working sensor nodes are well-distributed.

Proof: In ACHE protocol, every node must compete for working node with its neighboring node. Assume that after one node $v$ broadcasts an Active_Msg message within its transmission range $R t$, it can guarantee that all neighboring nodes can receive Active_Msg message from node $v$ in the interval $\Delta t$. Obviously, if no other neighboring nodes of node $v$ broadcast Active_Msg message in the interval $\Delta t$, node $v$ becomes the only one working node within its transmission range $R t$. On the other hand, if other neighboring nodes broadcast $\boldsymbol{A c}$ tive_Msg in the interval $\Delta t$, there maybe exist several working nodes within one transmission range Rt. Consider the probability that several working nodes within one transmission range $R t$, denoted as $p$.

According to (2), the time when any node broadcasts an Active_Msg message is approximately random uniformly distributed in $[0, T]$. Therefore, the probability $p$ could satisfy the Equation (6).

$$
p \leq 1-C_{m-1}^{0}\left(1-\frac{\Delta t}{T}\right)^{m-1}
$$

where $m-1$ is the expected value of the number of sensor nodes within the transmission range $R t$.

Clearly, $m=\left\lceil\frac{\pi R t^{2}}{\|A\|} \times N\right\rceil$. With typical values of other parameters, the probability $p$ is very small. For example, $R t=10 \mathrm{~m},\|\boldsymbol{A}\|=(100 \times 100) \mathrm{m}^{2}, N=100, T=10 \mathrm{~s}$, $\Delta t=10 \mathrm{~ms}$, the resulting $p=0.00214$.

Therefore, the probability that several working nodes within one transmission range $R t$ is small.

Theorem 4: ACHE has a worst case message exchange complexity of $O(1)$ per node and of $O(N)$ in the network.

Proof: During the execution of ACHE, if node $v$ re- ceived the Active_Msg messages from its neighbouring nodes before the time $t_{v}$ expires, node $v$ enters into the SLEEP state and does not send any message. Otherwise, node $v$ enters into the ACTIVE state and broadcasts an Active_Msg message. Since the number of these $\boldsymbol{A c}$ tive_Msg messages in the network is less than $N$, the number of messages exchanged in the network is upper-bound by $O(N)$.

\section{Simulation Study}

\subsection{Simulation Setup and Parameters}

To evaluate the performance of the ACHE protocol, we use GloMoSim [14] as the simulation platform in terms of evaluating the number of working nodes, coverage ratio, the network lifetime and control overhead. In the simulation, 600-1200 sensors are randomly deployed in a $100 \times 100\left(\mathrm{~m}^{2}\right)$ region, and each sensor has a sensing range of 10 meters, the maximum transmission range of 20 meters. To demonstrate ACHE could better adapt with the heterogeneous energy capacities in the sensor networks than others, simulation setups three different degree for heterogeneous energy among the sensor nodes. The initial energy of sensor nodes in the network is randomly uniformly distributed in $(0.2 \mathrm{~J}, 2.0 \mathrm{~J}),(1.0 \mathrm{~J}, 2.0 \mathrm{~J})$, and $(1.5 \mathrm{~J}, 2.0 \mathrm{~J})$ respectively. To evaluate the performance of energy efficiency, we use the same network parameters and radio model as presented in [15].

In article [3], we have compared DELIC protocol with other coverage protocols. DELIC protocol outperforms the Sponsor area [6], OGDC, PEAS in term of coverage ratio, energy efficiency and balancing energy load. Thus, we only compare proposed ACHE with DELIC in our simulation experiment.

\subsection{Effectiveness of $\mathrm{ACHE}$}

Figure 1 and Figure 2 give the curves of the number of working nodes and sensing coverage ratio in the different deployed nodes density with the different degree of heterogeneous energy capacities. As shown in Figure 1 and Figure 2, the number of working nodes obtained by ACHE is smaller about $15 \%$ than that of DELIC in the different nodes density. In the most situations, ACHE can provide above $99 \%$ sensing coverage for the different node density. But the quality of sensing coverage by ACHE is little poorer than that of DELIC. For the most application, the above $99 \%$ sensing coverage is acceptable and reasonable. In addition, as the same with DELIC, the number of working nodes by ACHE does not increase with the number of deployed nodes. This is because the number of working nodes in ACHE is related 


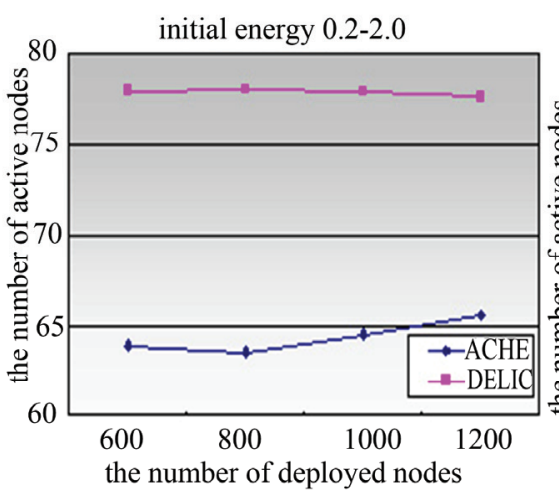

(a)

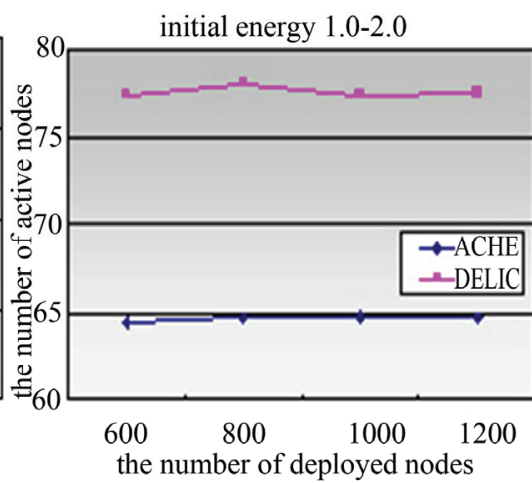

(b)

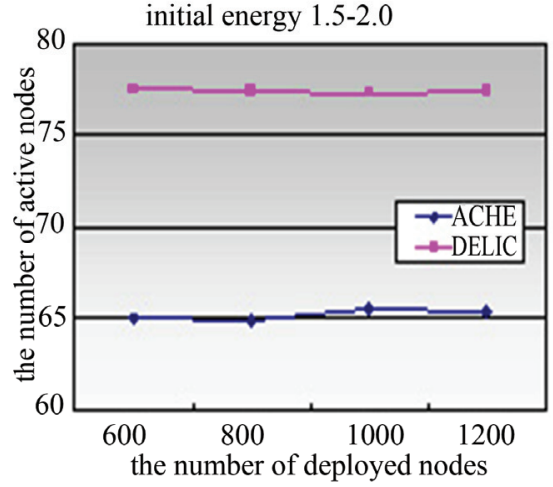

(c)

Figure 1. The number of active nodes in the different node density. (a) initial energy: $0.2 \mathrm{~J}-2.0 \mathrm{~J}$; (b) initial energy: $1.0 \mathrm{~J}-2.0 \mathrm{~J}$; (c) initial energy: $1.5 \mathrm{~J}-2.0 \mathrm{~J}$.

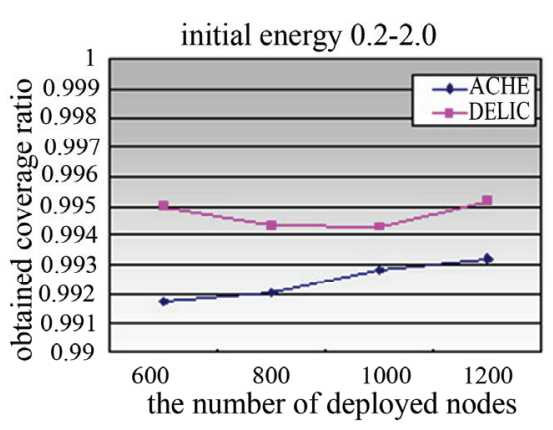

(a)

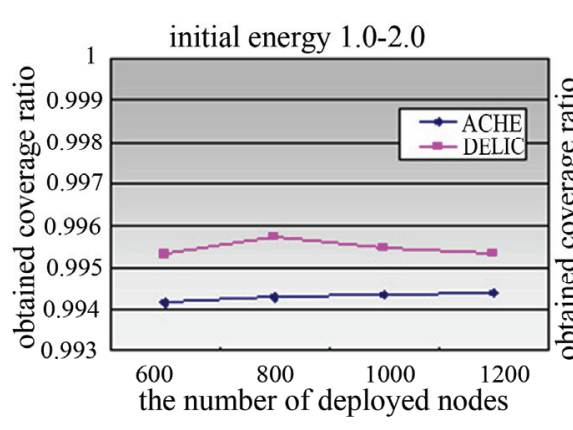

(b)

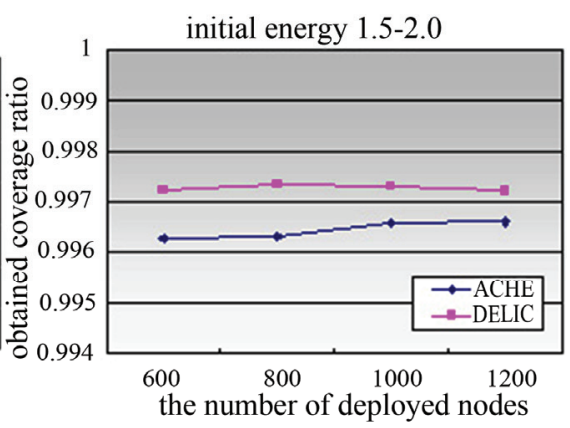

(c)

Figure 2. The sensing coverage ratio in the different node density. (a) initial energy: $0.2 \mathrm{~J}-2.0 \mathrm{~J}$; (b) initial energy: $1.0 \mathrm{~J}-2.0 \mathrm{~J}$; (c) initial energy: $1.5 \mathrm{~J}-2.0 \mathrm{~J}$.

with the sensing range of nodes, and the area of monitored region. The node density has no much impact on the number of working nodes. Figure 1 and Figure 2 demonstrate that ACHE can effectively provide the high quality of sensing coverage.

\subsection{Network Lifetime}

Figure 3 and Figure 4 illustrate the number of dead nodes with execution of ACHE in the scenario of deployed 600 nodes and 1200 nodes with the different degree of heterogeneous energy respectively. It can be seen that compared to DELIC, ACHE can effectively prolong the network lifetime by $15 \%-30 \%$ under the same situation. This is because DELIC protocol selects working nodes only considering the node its own residual energy, while ACHE also consider the current energy of neighboring nodes. Thus, ACHE protocol can better adapt the situation of the great heterogeneous energy capacities in the network. As Figure 3 and Figure 4 shown, when the initial energy of nodes vary from 0.2 to 2.0 , due to the great heterogeneous energy capacities, the speed of nodes' death is quicker $40 \%$ to $50 \%$ than that of the initial energy vary from 1.5 to 2.0 with the same network configuration.

If the current sensing coverage is lower than the threshold, sensor networks could not well perform the monitoring task. Thus, we evaluate the sensing coverage variation with the execution of ACHE. Figure 5(a) shows the curves of sensing coverage for ACHE with the three different degrees of initial energy when the deployed sensor nodes are 800 . It can be seen that the greater heterogeneous energy sensor nodes have, the quickly the sensing coverage drops. When the initial energy of sensor nodes vary from $0.2 \mathrm{~J}$ to $2.0 \mathrm{~J}$, ACHE could provide above $90 \%$ sensing coverage about 240 rounds. While the initial energy capacities of nodes vary from $1.5 \mathrm{~J}$ to $2.0 \mathrm{~J}$, ACHE could provide the same coverage ratio about 420 round. Meanwhile, the network lifetime can prolong about $30 \%$. In addition, Figure 5(b) illustrates the curves of sensing coverage for ACHE and DELIC with the same network configuration. It can be 


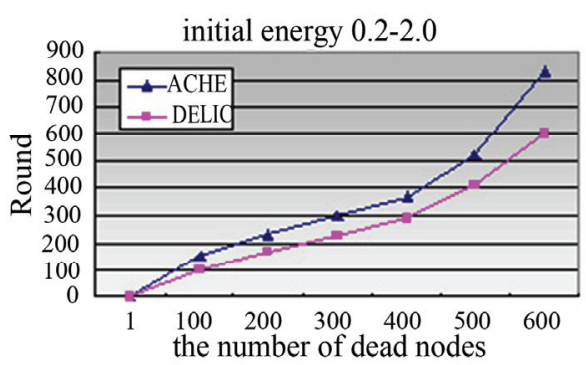

(a)

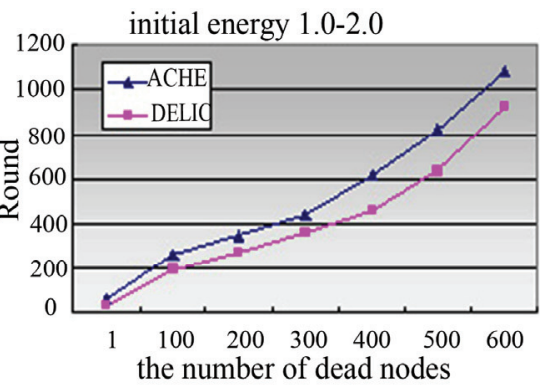

(b)

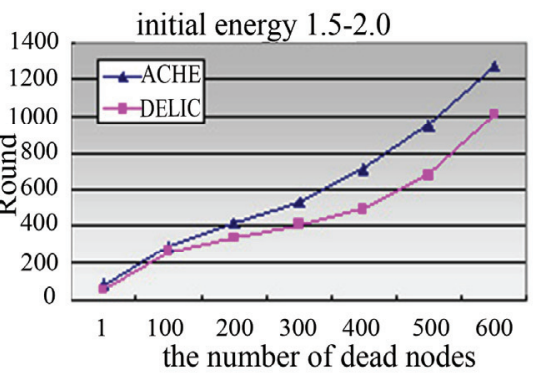

(c)

Figure 3. Timing of the nodes' death (600 nodes). (a) initial energy: $0.2 \mathrm{~J}-2.0 \mathrm{~J}$; (b) initial energy: $1.0 \mathrm{~J}-2.0 \mathrm{~J}$; (c) initial energy: $1.5 \mathrm{~J}-2.0 \mathrm{~J}$.

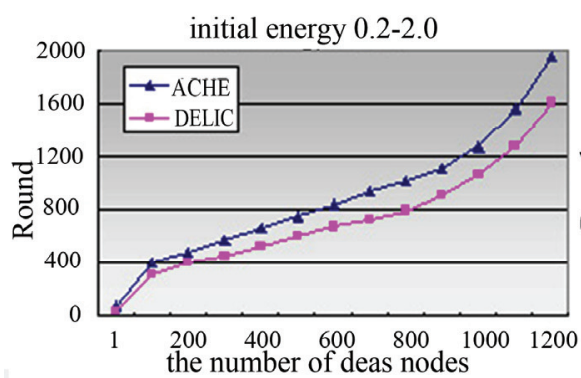

(a)

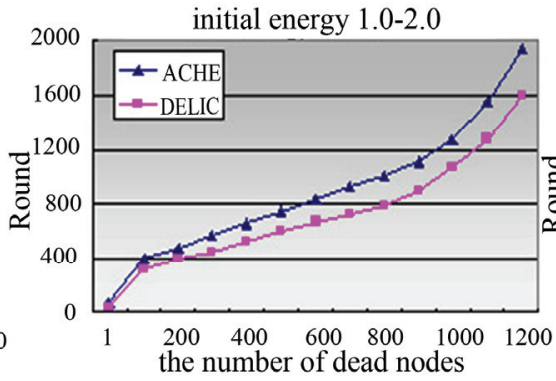

(b)

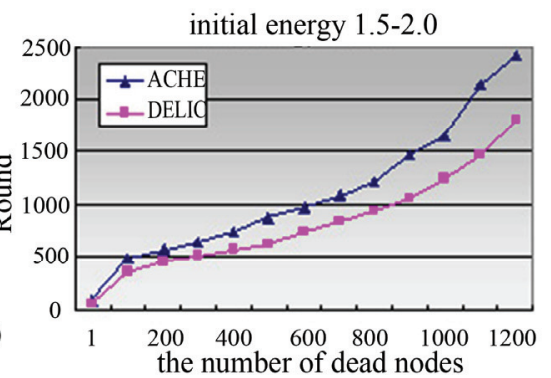

(c)

Figure 4. Timing of the nodes' death (1200 nodes). (a) initial energy: $0.2 \mathrm{~J}-2.0 \mathrm{~J}$; (b) initial energy: $1.0 \mathrm{~J}-2.0 \mathrm{~J}$; (c) initial energy: $1.5 \mathrm{~J}-2.0 \mathrm{~J}$.

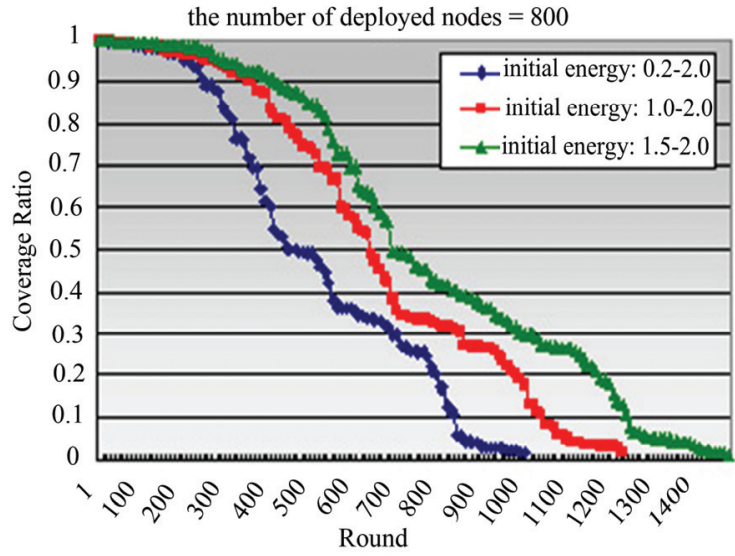

(a)

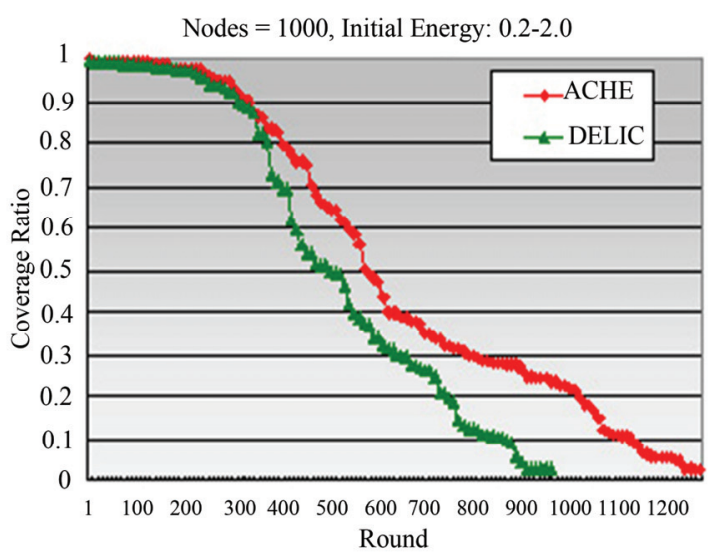

(b)

Figure 5. The sensing coverage ratio vs. round. (a) initial energy: $0.2 \mathrm{~J}-2.0 \mathrm{~J}$; (b) initial energy: $1.5 \mathrm{~J}-2.0 \mathrm{~J}$.

seen that the curve of sensing coverage of ACHE decreases more slowly than that in DELIC. This is due to the fact that ACHE can better adapt the heterogeneous energy capacities among senor nodes than DELIC.

\subsection{Control Overhead}

For ACHE, first, each nodes needs to count the number of 1-hop neighbors and adjust the appropriate transmission range. Thus, each node consumes energy to receive and send control messages. Second, in the phase of working nodes selection, every node also broadcasts an Energy_Msg message to its neighbor nodes to update the Neighbor_Table. Moreover, every working node needs to broadcast an Active_Msg message within its transmission range to announce its state. Therefore, the node density could have an impact on the control overhead.

Figure 6 compares the control overhead in the different node density with the different degrees of initial energy capacities between ACHE and DELIC. It is obvious 


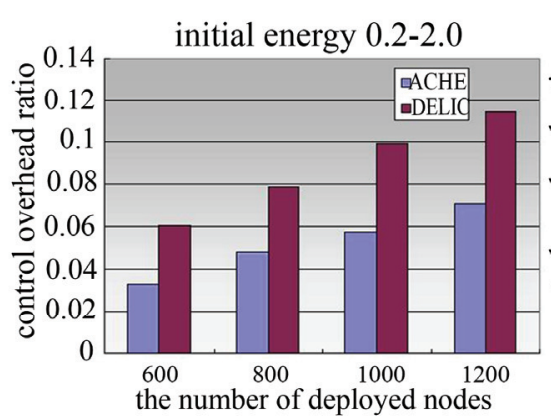

(a)

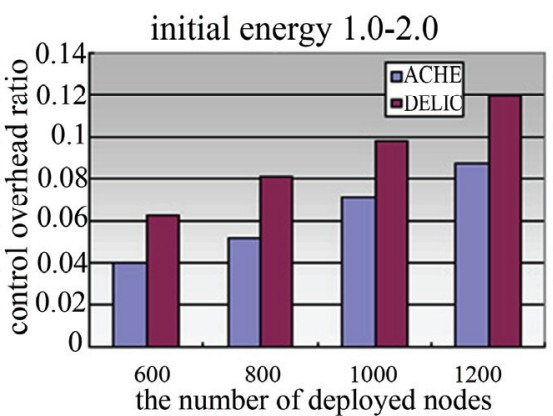

(b)

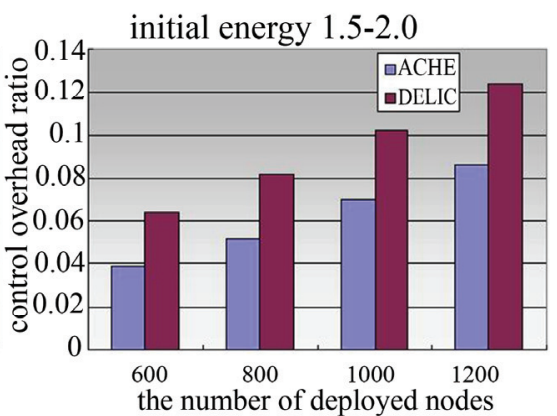

(c)

Figure 6. Control overhead in the different node density. (a) initial energy: 0.2 J-2.0 J; (b) initial energy: 1.0 J-2.0 J; (c) initial energy: $1.5 \mathrm{~J}-2.0 \mathrm{~J}$.

that the higher node density, the more control overhead in the same network configuration. In ACHE, the control overhead with 600 nodes is about twice that with 1200 nodes. On the other hand, it can be seen that the heterogeneous energy capacities in the network do not have great impact on the control overhead.

\section{Conclusions}

In this paper, an energy-aware location-independent coverage control protocol for heterogeneous wireless sensor networks (ACHE) is proposed. ACHE can achieve a good performance in terms of sensing coverage, lifetime by minimizing energy consumption for control overhead, and balancing the energy load among all nodes. Adopting the hierarchical clustering idea, ACHE elects the working nodes based on the average residual energy of neighboring nodes and its own residual energy parameters. Our simulation study and analysis demonstrate that ACHE not only provide the high quality of sensing coverage, but also has the good performance in the energy efficiency. In addition, ACHE can better adapt the applications with the great heterogeneous energy capacities in the sensor networks, as well as effectively reduce the control overhead.

\section{Acknowledgements}

This research is supported by the NSF of Hohai University under Grant No. 2008428911, and the Fundamental Research Funds for the Central Universities 2009B20714.

\section{References}

[1] I. F. Akyildiz, W Su, Y. Sankarasubramaniam and E. Cayirci, "Wireless Sensor Networks: A Survey," Computer Networks, Vol. 38, No. 4, 2002, pp. 393-422.

[2] I. Stojmenovic, "Position Based Routing in Ad Hoc Net- works," IEEE Communications Magazine, Vol. 40, No. 7, 2002, pp. 128-134.

[3] Y. C. Mao, M. Liu, L. J. Chen and D. X. Chen, "A Distributed Energy-Efficient Location-Independent Coverage Protocol in Wireless Sensor Networks," Computer Research and Development, Vol. 43, No. 2, 2006, pp. 187-195.

[4] S. Slijepcevic and M. Potkonjak, "Power Efficient Organization of Wireless Sensor Networks," Proceedings of IEEE Conference on Communications, Helsinki, 2001, Vol. 2, pp. 472-476.

[5] M. Cardei, D. MarCallum, X. Cheng, M. Min, X. Jia, D. Li, et al., "Wireless Sensor Networks with Energy Efficient Organization," Journal of Interconnection Networks, Vol. 3, No. 3-4, 2002, pp. 213-229.

[6] D. Tian and N. D. Georganas, "A Coverage-Preserving Node Scheduling Scheme for Large Wireless Sensor Networks," Proceedings of 1st ACM International Workshop on Wireless Sensor Networks and Applications, Atlanta, 2002, pp. 32-41.

[7] H. Zhang and J. C. Hou, "Maintaining Sensing Coverage and Connectivity in Large Sensor Networks," International Journal of Ad Hoc \& Sensor Wireless Networks, Vol. 1, No. 1-2, 2005, pp. 89-124.

[8] X. Wang, G. Xing, Y. Zhang, C. Lu, R. Pless and C. D. Gill, "Integrated Coverage and Connectivity Configuration in Wireless Sensor Networks," Proceedings of the 1st International Conference on Embedded Networked Sensor Systems, Los Angeles, 2003, pp. 28-39.

[9] T. T. Wu and K. F. Su, "Determining Active Sensor Nodes for Complete Coverage without Location Information," International Journal of Ad Hoc and Ubiquitous Computing, Vol. 1, No. 1-2, 2005, pp. 38-46.

[10] S. Kumar, T. H. Lai and J. Balogh, "On K-Coverage in a Mostly Sleeping Sensor Network," Proceedings of the 10th Annual International Conference on Mobile Computing and Networking, Philadelphia, 2004, pp. 144-158.

[11] F. Ye, G. Zhong, S. Lu and L. Zhang, "PEAS: A Robust Energy Conserving Protocol for Long-Lived Sensor Networks," Proceedings of the 23rd International Conference on Distributed Computing Systems, Providence, 2003, pp. 28-37. 
[12] K. Wu, Y. Gao, F. Li and Y. Xiao, "Lightweight Deployment-Aware Scheduling for Wireless Sensor Networks," ACM/Kluwer Mobile Networks and Applications, Vol. 10, No. 6, 2004, pp. 837-852.

[13] Y. C. Mao, G. F. Feng, L. J. Chen and D. X. Chen, "A Location-Independent Connected Coverage Protocol for
Wireless Sensor Networks," Journal of Software, Vol. 18, No. 7, 2007, pp. 1672-1684.

[14] http://pcl.cs.ucla.edu/projects/glomosim/

[15] W. Heinzelman, "Application-Specific Protocol Architecture for Wireless Sensor Networks," Ph.D. Thesis, Massachusetts Institute of Technology, Boston, 2000. 